A. Weisbecker, M. Burmester \& A. Schmidt (Hrsg.): Mensch und Computer 2015 Workshopband, Stuttgart: Oldenbourg Wissenschaftsverlag, 2015, S. 445-452.

\title{
Brain-Computer Interfaces als Arbeitsmittel der Zukunft? - Chancen und Grenzen aus Sicht eines Interaktionsgestalters
}

\author{
Kathrin Pollmann \\ Institut für Arbeitswissenschaft und Technologiemanagement, Universität Stuttgart
}

\section{Zusammenfassung}

In der zunehmend digitalisierten Arbeitswelt gewinnt die Gestaltung von Schnittstellen zwischen Mensch und technischem Arbeitsmittel zunehmend an Bedeutung. Brain-Computer Interfaces bieten eine Möglichkeit, die kognitiven Verarbeitungsprozesse und mentalen Zustände des Nutzers während der Interaktion mit technischen Systemen zu erfassen und diese zur Verfügung zu stellen. Mit Hilfe dieses zusätzlichen Informationskanals könnten sich die Systeme an individuelle Nutzerbedürfnisse und -anforderungen anpassen. In der vorliegenden Arbeit werden Chancen und Grenzen der Integration von Brain-Computer Interfaces in das Arbeitsumfeld für die Faktoren Mensch, Business, Technologie und Gesellschaft erläutert und Potenziale einer nutzerzentrierten Gestaltung dieser neuen Technologie diskutiert.

\section{Interaktionsgestaltung für die Arbeit von morgen}

In unserer zunehmend digitalisierten Arbeitswelt gewinnt das Forschungsfeld der MenschTechnik Interaktion (MTI) immer mehr an Bedeutung. Neue technische Arbeitsmittel werden verstärkt in den Arbeitsalltag integriert. Neben dem Ausbau von Desktoparbeitsplätzen, halten Roboter Einzug in die industrielle Fertigung, Ingenieure planen und entwickeln ihre Lösungen in 3D-Umgebungen und Entfernungen von mehreren tausend Kilometern werden mit Hilfe von virtuellen Kollaborationsumgebungen überbrückt.

Interaktionsgestaltern kommt im Rahmen dieser fortschreitenden technologischen Entwicklung die Aufgabe zu, Schnittstellen zwischen Nutzer und digitalen Arbeitsmitteln zu schaffen, die für den Nutzer intuitiv bedienbar sind. Neben einer effektiven und effizienten 
Aufgabenerledigung im Sinne einer guten Usability, spielen hierbei gerade am Arbeitsplatz auch zunehmend kognitive Nutzerfaktoren wie Emotionen, Werte und Belastung eine wichtige Rolle. Denn ein positives Nutzungserleben kann dazu beitragen, Produktivität, Motivation und Zufriedenheit der Mitarbeiter maßgeblich zu verbessern (Hassenzahl 2008; Spath et al. 2010).

Gleichzeitig werden durch den technischen Fortschritt neue Werkzeuge und Lösungsmittel für die Interaktionsgestaltung geschaffen. Der Trend geht klar zu neuen Eingabe- und Kommunikationsformen in der MTI. Die herkömmlichen Eingabewerkzeuge Tastatur und Maus werden zwar nach wie vor genutzt, an vielen Stellen aber schon durch TouchTechnologien abgelöst. Auch Gesten und Sprache können mittlerweile relativ zuverlässig genutzt werden, um Maschinen zu steuern. Bald auch unsere Gehirnströme?

\section{Mensch-Technik Interaktion mittels BCI}

Aktuell geht der Trend dahin, Brain-Computer Interfaces (BCIs) aus ihrem ursprünglichen medizinischen Anwendungsfeld zu verlagern und für gesunde Probanden zu erschließen. In diesem Zusammenhang gilt es zu untersuchen, inwieweit BCIs für die MTI nutzbar gemacht werden können.

Kognitionswissenschaftler postulieren, dass soziale Interaktion und Kommunikation mit anderen Menschen vor allem deswegen funktioniert, weil wir ein mentales Modell bilden, das den aktuellen Zustand der anderen Person nachbildet (vgl. „Theory of Mind“, z.B. in Premark \& Woodruff 1978, und „Simulation Theory“, z.B. in Goldman 1992). Dies ermöglicht uns, Handlungsintentionen unseres Gegenübers automatisch und in Sekundenbruchteil zu erkennen und unsere Handlungen entsprechend anzupassen (Cuijpers et al. 2006). Diese Fähigkeit fehlt technischen Systemen bislang gänzlich, denn aktuelle Modelle und Sensoren sind nicht sensibel genug, um kognitive Zustände ihrer menschlichen Interaktionspartnern zu erfassen (Zander et al. 2010). Die Schlussfolgerung liegt also nahe, dass die MTI entscheidend verbessert werden kann, wenn dem technischen System Informationen über den aktuellen mentalen Zustand des menschlichen Interaktionspartners mittels eines BCI zur Verfügung gestellt werden. Grundsätzlich sind in diesem Zusammenhang zwei interessante Anwendungsszenarien denkbar:

Zum einen können BCIs genutzt werden, um eine aktive Ansteuerung von technischen Systemen durch Gehirnaktivität zu ermöglichen (aktives BCI, Zander et al. 2008). BCIs wurden in der medizinischen Forschung ursprünglich für Patienten mit neuromuskulären Erkrankungen wie Amyotropher Lateralsklerose oder Wirbelsäulenverletzungen entwickelt (Birbaumer et al. 1999; Kübler et al. 2005). Diese Menschen können ihre Muskeln nicht mehr kontrollieren, die höheren kognitiven Funktionen bleiben dabei aber intakt. Für sie bieten aktive BCIs eine Möglichkeit der Kommunikation mit der Außenwelt. In ausgiebigen Trainingssessions lernen die Patienten, bewusst bestimmt Hirnsignale hervorzurufen und zu kontrollieren. Auch in der MTI könnten solche bewusst erzeugten Hirnsignale als neuer 
Informationskanal eingesetzt werden, um direkt mit technischen Systemen zu kommunizieren.

Zunehmend an Attraktivität gewinnt außerdem die Idee, BCIs zur passiven Überwachung der mentalen Zustände des Nutzers einzusetzen (passives BCI; Zander et al. 2008). Den Maschinen könnten so ohne explizite kognitive Anstrengung des Nutzers wichtige Informationen z.B. über momentane Intentionen, Emotionen, Aufmerksamkeitszustände oder kognitive Belastungen des Nutzers übermittelt werden. Ein System, das entsprechende adaptive Funktionalitäten umfasst, könnte seine Benutzeroberfläche und Funktionen vor Ort und in Echtzeit immer wieder iterativ an den individuellen Nutzer angepasst werden, was die Interaktion nachhaltig verbessern würde.

Grundsätzliche Visionen zur Nutzbarmachung von BCIs für die MTI im Allgemeinen und den Einsatz im Arbeitsalltag im Speziellen sind also vorhanden. Es bleibt allerdings zu untersuchen, inwieweit diese Ideen zum jetzigen Zeitpunkt bereits umsetzbar sind.

\section{Chancen und Grenzen des BCI}

Bei der Einschätzung des Erfolgs eines innovativen Produkts werden im menschzentrierten Ansatz des Design Thinking drei Faktoren herangezogen, deren Schnittmenge das Erfolgspotenzial des Produkts widerspiegelt (vgl. Abbildung 1): Von großer Bedeutung ist der Mensch mit seinen Wünschen und Bedürfnissen. Aber ob ein Produkt tatsächlich das Potenzial hat, ein Erfolg zu werden, hängt auch von seiner technischen Umsetzbarkeit und Rentabilität ab. Wenn es um die Messung von Gehirnströmen geht, spielt zudem der gesellschaftliche Rahmen inklusive ethischen, rechtlichen und politischen Fragestellungen eine entscheidende Rolle dafür, ob das Produkt Marktreife erreicht.

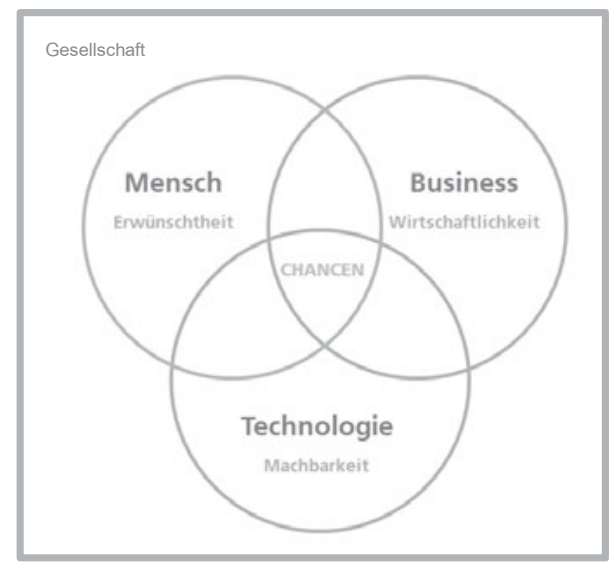

Abbildung 1. Chancen für das BCI als Innovation in der Arbeitswelt (in Anlehnung an Kelley \& Kelley 2013) 
Um den tatsächlichen Mehrwert einer BCI-Anwendung als Schnittstelle für digitale Arbeitsmittel zu bewerten, gilt es zunächst ihre Potenziale und Grenzen bezüglich der genannten Faktoren herauszustellen.

\subsection{Technologie - was ist möglich?}

Zur Umsetzung eines BCI in einer natürlichen Interaktionsumgebung wie dem Arbeitsplatz werden Technologien benötigt, die nicht-invasiv, kostengünstig, mobil und sicher sind (Tan \& Nijhold 2010). Diese Kriterien werden momentan nur von zwei neurowissenschaftlichen Messverfahren erfüllt: Vorrangig wird zur Umsetzung von BCIs die Elektroenzephalographie (EEG) verwendet, mit deren Hilfe die elektrische Aktivität im Kortex erfasst werden kann. Alternativ (oder ergänzend) hat sich aber die funktionelle Nahinfrarotspektroskopie (fNIRS), als geeignetes Verfahren zur Erfassung von Hirnaktivität erwiesen, wobei diese indirekt über den Sauerstoffgehalt im Blut abgeleitet wird. Beide Verfahren erscheinen grundsätzlich zum Einsatz während der MTI geeignet, der Einsatz außerhalb von Laborräumen ist aber aktuell noch nicht uneingeschränkt möglich.

Die Mobilität dieser Technologien wurde in den letzten Jahren stetig erhöht und die Tendenz geht zu einer weiteren Miniaturisierung der Hardware, insbesondere der kopfgetragenen Messeinheit, um die Nutzbarkeit für Anwendungsgebiete außerhalb der Medizintechnik zu steigern (z. B. De Vos et al. 2014). Inwieweit die BCI-Sensorik aber letztendlich verkleinert werden kann, hängt neben der technischen Machbarkeit auch von der Verortung der kognitiven Verarbeitungsprozessen ab, die erfasst werden sollen.

Bei der Leistungsfähigkeit der Geräte scheint noch Verbesserungsbedarf zu bestehen. Bei Labor-Untersuchungen sind die Geräte immer nur über Zeiträume von wenigen Stunden in Betrieb. Wie sich ein Langzeitgebrauch über einen kompletten Arbeitstag hinweg realisieren lässt, muss noch untersucht werden. Auch die Länge der Kalibrierungszeiten erschwert den Einsatz am Arbeitsplatz: Beim BCI fallen derzeit für die Kalibrinerung 5-20 Minuten an (Blankertz et al. 2006).

Auch die Echtzeitanalyse der Daten, ohne die eine sofortige Berücksichtigung der erfassten Hirnzustände in der Interaktionsgestaltung nicht realisieren lässt, ist noch nicht endgültig gelöst. In diesem Zusammenhang müssen auch Lösungen für die Reduzierung von Bewegungsartefakten erarbeitet werden, für die gerade das EEG-Signal sehr anfällig ist, die sich aber bei der aktiven Arbeit kaum vermeiden lassen (Fatourechi et al. 2007).

\subsection{Business - was ist profitabel?}

Im Rahmen der Digitalisierung werden Prozesse in Unternehmen aus fast allen Branchen zunehmend automatisiert. Zum aktuellen Zeitpunkt können Maschinen die Mitarbeiter allerdings noch nicht komplett ersetzen, sondern nehmen ihnen Teilaufgaben ab oder arbeiten ihnen zu. Der Mensch stellt auch weiterhin eine wichtige Ressource für das Unternehmen dar - insbesondere weil Wissensarbeit in allen Branchen immer wichtiger wird. Das Unternehmen hat daher durchaus ein großes Interesse daran, die Gesundheit, 
Arbeitsfähigkeit, Produktivität und Innovationsfähigkeit der Mitarbeiter über einen langen Zeitraum hinweg zu erhalten und zu stärken.

BCI-Anwendungen haben das Potenzial, diesen Prozess zu unterstützen: Wie hoch die kognitive Belastung bei der Bearbeitung einer oder mehrere Aufgabe(n) ist, kann der Nutzer selbst oft nicht richtig einschätzen. Ein System, das über die Hirnsignale des Nutzers seinen Belastungszustand ableiten kann, könnte Überbeanspruchungen direkt entgegenwirken und z.B. den Nutzer über seinen Zustand informieren und entlastende Maßnahmen vorschlagen oder eine automatische Anpassung des Systemverhaltens initiieren. Auch Informationen über kognitive Prozesse wie emotionales Erleben oder Aufmerksamkeit können genutzt werden, um die Interaktionsgestaltung noch während der MTI nutzerzentriert zu verbessern, dadurch Stresssituation zu reduzieren und Wohlbefinden und Resilienz zu erhöhen.

Zur Einschätzung des tatsächlichen Mehrwerts des Einsatzes von BCIs im Arbeitsalltag fehlen aber momentan noch messbare Erfolgsgrößen. Eine Verbesserung des Zustands der Mitarbeiter wirkt sich kurzfristig nicht sichtbar auf den Unternehmenserfolg aus. Hier müssten Langzeitstudien durchgeführt werden, um eine signifikante Verbesserung aufzuzeigen, die dann auch das Argument für eine dauerhafte Integration von BCI-Systemen mit digitalen Arbeitsmitteln liefern könnte. Die aktuell verfügbaren Technologien sind zwar im Vergleich zu anderen Verfahren wie der Magnetresonanztomographie (MRT) relativ kostengünstig, aber trotzdem eine große Investition, wenn viele Geräte angeschafft werden sollen. Bei den hohen Anschaffungskosten und dem noch genau zu bestimmenden Nutzen für Unternehmen, scheint die Integration von BCIs in den Arbeitsalltag zum jetzigen Zeitpunkt also wenig profitabel. Es stellt sich außerdem die Frage, ob vergleichbare Ergebnisse nicht auch mit preisgünstigeren Lösungen erzielt werden können.

\subsection{Mensch - was ist erwünscht?}

Nicht zuletzt entscheiden die Bedarfe und Wünsche des Nutzers darüber, ob ein Produkt die Marktreife erreicht. Die Schaffung eines ,gesünderen“ Arbeitsplatzes mit Arbeitsmitteln, die sich an den individuellen Nutzeranforderungen orientieren, stellt durchaus eine erstrebenswerte Situation für die Beschäftigten dar. Allerdings gilt es abzuwägen, ob dieser Nutzen höher ist, als der Aufwand und die Einschränkungen, die mit Nutzung eines BCIs einhergehen.

Zum einen, ist der Trainingsaufwand für das Einlernen eines aktiven BCIs sehr hoch. Gelähmte Patienten haben in der Regel die Zeit und Motivation, die langwierigen Trainingssessions durchzuführen, allerdings ist für sie der Mehrwert eines erfolgreichen Einlernens auch unverhältnismäßig höher. Darüber hinaus hat sich gezeigt, dass für ca. $20 \%$ der Bevölkerung die Erkennungsgenauigkeit selbst durch Training nicht ausreichend gesteigert werden kann, um eine aktive Ansteuerung durch das BCI zu ermöglichen (,BCI illiteracy“; Tan \& Nijhold 2010; Blankertz et al. 2010). Bei passiven BCIs ist kein intensives Training nötig, weshalb sie für den Einsatz mit gesunden Nutzern etwas besser geeignet erscheinen. 
Die Gestaltung der Hardware und insbesondere der Sensorik stellt eine weitere potenzielle Nutzungsbarriere dar. Zur Nutzung des BCI müssen Sensoren am Kopf angebracht werden. EEG- und fNIRS-Systeme, die in der Forschung eingesetzt werden, verwenden hierzu in der Regel eine Kappe, die den gesamten Kopf des Nutzers abdeckt. Es gibt allerdings auch bereits andere, teils kommerzielle Lösungen, die gezielt Signale in einzelnen Hirnregionen messen und eine entsprechend miniaturisierte Hardware dazu bereitstellen, z.B. in Form eines Headset (z.B. Emotiv EPOCH; www.emotive.de). Für die Zukunft ist durchaus denkbar, die BCI-Sensorik in andere kopfgetragene Hardware, die in bestimmten Berufsfeldern standardmäßig getragen wird (z.B. Arbeitshelme), zu integrieren und so möglichen Akzeptanzproblemen entgegenzuwirken.

\subsection{Gesellschaft - was ist ethisch, politisch, rechtlich vertretbar?}

Wie auch anderen sensorbasierten Technologien greifen BCI-Systeme auf Nutzerdaten zu. Die Gesellschaft muss sich also, wenn es um den Einsatz des BCI in der Arbeitswelt geht, mit Fragen der Erhebung, Speicherung und Löschung personenbezogener Daten auseinandersetzen und die Regierung den entsprechenden politischen und rechtlichen Rahmen schaffen (Stopczynski et al. 2014). Insbesondere gilt es zu diskutieren ob sichergestellt werden kann, dass Nutzer, die ein passives BCI verwenden, bei dem sie nicht standardmäßig direktes Feedback über ihren mentalen Zustand bekommen, trotzdem weiterhin die volle Kontrolle über ihre Daten innehaben.

\section{Fazit und Ausblick}

Insgesamt steckt die Entwicklung von BCIs für anwendungsorientierte MTI-Szenarien noch in ihren Kinderschuhen. Aktuell ist eine technische Grundbasis vorhanden, die solide, aber ausbaufähig ist. Insbesondere die Anschaffungskosten und der Aufwand für die Inbetriebnahme eines BCI sind aktuell noch sehr hoch. Hinzu kommt, dass, im Gegensatz zum medizinischen Anwendungsgebiet, der Mehrwert für das Arbeitsumfeld nicht unmittelbar messbar und daher noch fraglich ist. Auch die Nutzerakzeptanz ist aufgrund des aktuellen Nutzungsaufwands und der Hardwaregestaltung noch steigerungsfähig. In der BCIForschung wird momentan, wie so oft bei technischen Innovationen, noch zu wenig menschzentriert gearbeitet. Die Übertragung der BCI-Technologien aus dem medizinischen Kontext auf andere Anwendungsfelder, in diesem Fall die Arbeitswissenschaft, ist nach wie vor stark technikgetrieben. Im Fokus steht dabei die Frage, wie man mit einer existierenden Technologie einen Mehrwert für das jeweilige Anwendungsfeld schaffen kann. Um die Chancen einer erfolgreichen Integration des BCI in die Arbeitsumgebung zu erhöhen, sollte in Zukunft verstärkt ein menschzentrierter Ansatz verfolgt werden, der zunächst Bedarfe der zukünftigen Nutzer analysiert und die Gestaltung der technischen Innovation daran ausrichtet. Das noch sehr junge Forschungsfeld des BCI bietet in diesem Punkt noch viel Gestaltungsspielraum und Möglichkeiten, den Nutzer in den Gestaltungsprozess miteinzubinden. 


\section{$5 \quad$ Schlussteil}

\section{Kontaktinformation}

Kathrin Pollmann

Nobelstr. 12, D-70569 Stuttgart

Email: kathrin.pollmann@iat.uni-stuttgart.de

Website: www.hci.iao.fraunhofer.de

Telefon: +497119702347

\section{Literaturverzeichnis}

Birbaumer, N., Ghanayim, N., Hinterberger, T., Iversen, I., Kotchoubey, B., Kübler, A., Perelmouter, J., Taub, E. \& Flor, H. (1999). A spelling device for the paralysed. Nature 398, 297-298.

Blankertz, B., Dornhege, G., Krauledat, M., Müller, K. R., Kunzmann, V., Losch, F. \& Curio, G. (2006). The Berlin Brain-Computer Interface: EEG-based communication without subject training. IEEE Transactions on Neural Systems and Rehabilitation Engineering 14(2), 147-152.

Blankertz, B., Sannelli, C., Halder, S., Hammer, E.M., Kübler, A., Müller, K.-R., Curio, G. \& Dickhaus, T. (2010). Neurophysiological predictor of SMR-based BCI performance. NeuroImage 51, 1303-1309.

Cuijpers, R. H., Schie, H. T. van, Koppen, M., Erlhagen, W. \& Bekkering, H. (2006). Goals and means in action observation: A computational approach. Neural Networks 19(3), 311-322.

De Vos, M., Gandras, K. \& Debener, S. (2014). Towards a truly mobile auditory brain-computer interface: exploring the P300 to take away. International journal of psychophysiology 91(1), 46-53.

Fatourechi, M., Bashashati, A., Ward, R. K. \& Birch, G. E. (2007). EMG and EOG artifacts in brain computer interface systems: A survey. Clinical neurophysiology 118(3), 480-494.

Goldman, A. I. (1992). In defense of the simulation theory. Mind \& Language 7(12), 104-119.

Hassenzahl, M. (2008). User Experience (UX): Towards an experiential perspective on product quality, In: IHM '08: Proceedings of the 20th French-speaking conference on Human-computer interaction.

Kelley, T. \& Kelley, D (2013). Creative Confidence: Unleashing the creative potential within us all. London, UK: William Collins.

Kübler, A., Nijboer, F., Mellinger, J., Vaughan, T.M., Pawelzik, H., Schalk, G., McFarland, D.J., Birbaumer, N. \& Wolpaw, J.R. (2005). Patients with ALS can use sensorimotor rhythms to operate a brain-computer interface. Neurology 64, 1775-1777.

Premack, D., \& Woodruff, G. (1978). Does the chimpanzee have a theory of mind? Behavioral and Brain Sciences, 1(04), 515-526.

Spath, D., Peissner, M., \& Sproll, S. (2010). Methods from neuroscience for measuring user experience in work environments. In: Proceedings of the International Conference on Applied Human Factors and Ergonomics (AHFE'10). 
Stopczynski, A., Greenwood, D., Hansen, L. K., \& Pentland, A. (2014). Privacy for Personal Neuroinformatics. Available at SSRN 2427564.

Tan, D., \& Nijholt, A. (2010). Brain-computer interfaces and human-computer interaction. In Tan, D. \& Nijholt, A. (Hrsg.): Brain-Computer Interfaces. London: Springer. [S.3-19].

Zander, T. O., Kothe, C., Welke, S. \& Roetting, M. (2008). Enhancing human-machine systems with secondary input from passive brain-computer interfaces. In: Proceedings of the 4th International BCI Workshop \& Training Course, Graz, Austria, 2008. Graz University of Technology Publishing House, Graz.

Zander, T. O., Kothe, C., Jatzev, S., \& Gaertner, M. (2010). Enhancing human-computer interaction with input from active and passive brain-computer interfaces. In Tan, D. \& Nijholt, A. (Hrsg.): Brain-Computer Interfaces. London: Springer. [S. 181-199]. 